\title{
Antibiotic use in Departments of Internal Medicine of Lazio
}

\author{
Dario Martolini, ${ }^{1}$ Maurizia Galiè, ${ }^{1}$ Anna Maria Santoro, ${ }^{2}$ Danilo Monno, ${ }^{3}$ Claudio Santini, ${ }^{1}$ David Terracina ${ }^{2}$ on behalf \\ of FADOI Lazio - Area of Infectious Diseases
}

${ }^{1}$ Department of Internal Medicine, Madre Giuseppina Vannini Hospital, Figlie di San Camillo Institute, Roma; ${ }^{2}$ Department of Internal Medicine, Sandro Pertini Hospital, Roma; ${ }^{3}$ Department of Internal Medicine, Fatebenefratelli Hospital, Isola Tiberina, Roma, Italy

\begin{abstract}
Antimicrobial therapy is inappropriate in 9 to $64 \%$ of the patients hospitalized. We evaluated the antibiotic use in Internal Medicine wards of an Italian region (Lazio) by a prospective multicenter, observational study. One thousand and nine patients were evaluated. Patients under antimicrobial treatment (PUAT) were 588 (58.2\%), patients without treatment (PWT) 421 (41.8\%). Infections were classified as community acquired (47.8\%), hospital acquired (10.3\%) or healthcare-associated (11.4\%); the remaining $30.5 \%$ of infections did not receive any epidemiological classification. Samples for microbiological examination were collected in $41.6 \%$ of PUAT. The antibiotic choice was empiric in $94.8 \%$ of the cases and protected penicillins were selected in $48 \%$ of the cases. The mean duration of treatment was $9.5 \pm 6$ standard deviation (SD) days. Only $6 \%$ of the patients switched from intravenous to oral therapy. Age, length of hospital stay and mortality were higher for PUAT than for PWT (mean age: $75.9 \pm 15 \mathrm{SD}$ s $74.2 \pm 15 \mathrm{SD}$ years, $\mathrm{P}<0.02$; length of hospital stay: $13.7 \pm 10.4 \mathrm{SD}$ vs $10 \pm 8.4 \mathrm{SD}$ days, $\mathrm{P}<0.01$; mortality: $15.9 \%$ vs 3.1\%). Antibiotic stewardship needs to be implemented all over the hospitals of Lazio region.
\end{abstract}

\section{Introduction}

Antibiotics are frequently prescribed in medical wards, representing among others, a considerable burden. Antimicrobial therapy and prophylaxis in hospi-

Correspondence: Dario Martolini, Department of Internal Medicine, Madre Giuseppina Vannini Hospital, Figlie di San Camillo Institute, via Monte Grimano 73, 00138 Roma, Italy.

E-mail: martolini.dario@yahoo.it

Key words: Antibiotic consumption; antibiotic stewardship; infectious diseases.

Collaborators: Aldo Fierro, Eleonora Pistella, Filomena Pietrantoni, Gianluca Pavoni, Dario Manfellotto, Rosa Maida, Gianfranco Panetta, Francesco Montella, Alessandro Bozza, Paolo Carfagna, Agapito Tarasi, Ruggero Pastorelli, Rosalba Cipriani, Basilio Battisti, Giovanna Giacchetti, Giuseppe Campagna, Cecilia Cianfrocca, Bruno Mongiardo, Alessandra Fiorentini (for the List of Centers see Table 1).

Conflict of interest: the authors declare no potential conflict of interest.

Received for publication: 2 December 2016.

Revision received: 14 July 2017.

Accepted for publication: 19 July 2017.

This work is licensed under a Creative Commons Attribution NonCommercial 4.0 License (CC BY-NC 4.0).

CCopyright D. Martolini et al., 2017

Licensee PAGEPress, Italy

Italian Journal of Medicine 2017; 11:364-370

doi:10.4081/itjm.2017.814 tals have been reported to be incorrect or not indicated in 9 to $64 \%$ of the patients ${ }^{1}$ and a similar problem is described also in other settings such as nursing homes where, due to the lack of structural and human resources, antimicrobial prophylaxis is often used in an attempt to prevent infections. ${ }^{2}$ There are several reasons for evaluating the treatment as inappropriate: lack of evidence of active bacterial disease, empiric treatment of non-critical patients, treatment of colonized asymptomatic patients, selection of second-line antibiotics not microbiologically driven. The main consequences of excessive or inappropriate antimicrobial use of antibiotics is the increase in infection or colonization due to resistant strains (methicillin resistant Staphylococcus aureus, multi-drug resistant Gramnegative bacteria, Clostridium difficile and Candida); these infections are burdened with high mortality, prolonged hospitalization and a substantial increase in health care costs. Moreover, antimicrobial drug toxicity and drug interactions should also be computed.

The main goal of antimicrobial stewardship is to obtain the best clinical outcome minimizing toxicity and limiting the selective pressure on bacterial populations that drives the emergence of resistant strains. The programs of antimicrobial stewardship improve the appropriate use of antimicrobial agents promoting the selection of the optimal antimicrobial regimen, including dosing, duration of therapy and route of administration. Antimicrobial stewardship also reduces the costs attributable to suboptimal antimicrobial use. Quantitative monitoring of antimicrobial use in hospitals or hospital units and qualitative evaluation of 
antimicrobial treatment and prophylaxis in individual patients are pivotal in addressing the interventions. ${ }^{3-5}$ Based on these premises we were interested in evaluating the modality of use of antibiotics in the wards of Internal Medicine of several hospitals of Lazio (an Italian region), focusing on number of treated patients, appropriateness of indications, preferred antibiotic classes, and followed stewardship programs (i.e., deescalation and/or rotation of antimicrobials).

\section{Materials and Methods}

Twelve units of Internal Medicine from 9 hospitals spread throughout the Italian region of Lazio participated in this prospective, multicenter, observational study performed on behalf of FADOI Lazio - Area of Infectious Diseases. The protocol was approved by the Local Ethic Committee. Every unit nominated a referent physician for data collection. The study period was 12 months (from November 2014 to October 2015). The list of participating hospitals, with the names of unit directors and referent physicians are reported in Table 1. Every patient, or their relatives when legally in charge, signed an informed consent form before any datum was collected. A data record form of every patient discharged during the first week of every month was filled. Every month all record forms were sent to the coordination center in order to create a single database. All collected patients were divided into two main groups: patients who underwent antimicrobial treatment (PUAT) and patients without treatment (PWT). These two populations were compared in terms of age, length of hospital stay, and mortality. For the PUAT group, the reasons to start an antimicrobial treatment and the ward or department where it was started were recorded. The diagnosis of in- fection was considered as possible, probable or definite according to diagnostic criteria guidelines and to the judgement of the doctor in charge. Every infection was classified for the organ or site affected, but also epidemiologically, discriminating among community-acquired (CAI), hospital-acquired (HAI) and healthcare-associated infections (HCAI). Inflammatory markers as procalcitonin (PCT) and/or C-reactive protein (CRP) and microbiological examinations performed were also recorded for all patients in the PUAT group. The antimicrobial therapy was evaluated if targeted on a specific isolate or empiric, and modifications in terms of upgrade or downgrade following microbiological isolation were reported. The used antimicrobials, the route of administration and the length of therapy were also considered. According to the clinical and epidemiological diagnosis, the appropriateness of the antibiotic chosen was evaluated from two different authors.

\section{Statistical analysis}

Data are expressed as mean \pm standard deviation (SD). Data were investigated if normally or not normally distributed. Non-parametric Mann-Whitney's test was used to compare two groups of non-parametric data. Kruskal-Wallis' test was employed to compare several groups of parametric data. GraphPad Prism 5.0 (GraphPad Software, Inc. USA) was used for statistical analysis.

\section{Results}

From November 2014 to October 2015, in the first week of every month, 1009 patients (M/F, 515/494; mean age/range 75.2 years $\pm 15.2 \mathrm{SD}$ ) were discharged

Table 1. Participating Centers and Units.

\begin{tabular}{|c|c|c|}
\hline Hospital & Unit-Ward Director(s) & Investigator(s) \\
\hline Vannini - Roma (coordinator center) & Claudio Santini & $\begin{array}{c}\text { Maurizia Galiè } \\
\text { Dario Martolini } \\
\text { Eleonora Pistella }\end{array}$ \\
\hline Sandro Pertini - Roma & $\begin{array}{c}\text { Aldo Fierro } \\
\text { David Terracina }\end{array}$ & Anna Maria Santoro \\
\hline Sant'Eugenio - Roma & Filomena Pietrantoni & Gianluca Pavoni \\
\hline Fatebenefratelli Isola Tiberina - Roma & Dario Manfellotto & Danilo Monno \\
\hline San Giovanni - Roma & $\begin{array}{c}\text { Rosa Maida } \\
\text { Gianfranco Panetta } \\
\text { Francesco Montella }\end{array}$ & $\begin{array}{c}\text { Alessandro Bozza } \\
\text { Paolo Carfagna } \\
\text { Agapito Tarasi }\end{array}$ \\
\hline L. Parodi Delfino - Colleferro & Ruggero Pastorelli & Rosalba Cipriani \\
\hline San Camillo de Lellis - Rieti & Basilio Battisti & Giovanna Giacchetti \\
\hline Santa Maria Goretti - Latina & Giuseppe Campagna & Cecilia Cianfrocca \\
\hline Montefiascone & Bruno Mongiardo & Alessandra Fiorentini \\
\hline
\end{tabular}


from the twelve Internal Medicine Units participating in the study and were evaluated. Out of them, $95 \%$ were addressed to the wards of Internal Medicine from the Emergency Department, 3\% from other wards, 1.2\% directly from community. PUAT group included 588 patients (58.2\%) and PWT group 421 patients (41.8\%). In 222 cases $(37.7 \%)$ the antibiotic therapy was initiated at the Emergency Department and in 339 cases (57.6\%) during the permanence in the Internal Medicine ward. The antibiotic choice was empiric in $94.8 \%$ of patients and culture-driven in $4.9 \%$ of patients. The reported reasons to start the antibiotic therapy were: definite infection in 245 patients (41.6\%), probable infection in 93 patients (15.8\%), possible infection in 97 patients (16.4\%), fever in $50(8.5 \%)$ and prevention in 103 patients (17.5\%). The infections were classified as CAI and $\mathrm{HAI}$ in $47.8 \%$ and $10.3 \%$ of patients respectively and HCAI in $11.4 \%$. The remaining $30.5 \%$ did not receive any epidemiological classification by the doctors in charge. The site of infection was available for 485 patients (Table 2). Mean age, length of stay and mortality were higher for PUAT compared to PWT [mean age PUAT vs PWT: $75.9 \pm 15$ SD vs $74.2 \pm 15$ SD years, $\mathrm{P}<0.02$; length of hospital stay PUAT $v s$ PWT: $13.7 \pm 10.4$ SD vs $10 \pm 8.4$ SD days, $\mathrm{P}<0.01$; mortality
PUAT vs PWT: 16.1\% (95 deaths) vs 3.1\% (13 deaths)]. CRP, PCT, or both were measured respectively in $72 \%$, $26 \%$, and $21 \%$ of PUAT. In the PUAT group, microbiological cultures were performed in 245 patients $(41.6 \%)$ and $121(49.3 \%)$ resulted positive. On the basis of culture isolates, empiric antibiotic regimens resulted inadequate in 41 patients (33.8\%); no modification was suggested in the remaining 80 patients $(66.1 \%)$. The change in antibiotic treatment was an escalation in 25 patients, a de-escalation in 9 patients, while 7 patients did not receive any change even if suggested by the culture results. Overall, among patients of the PUAT group, the antibiotics more frequently selected for empiric treatment were ampicillin-sulbactam (133 patients $=27.4 \%$ ), ceftriaxone $(102$ patients $=21 \%)$, fluoroquinolones $(92$ patients $=18.9 \%)$ and piperacillin-tazobactam $(81 \mathrm{pa}-$ tients $=16.7 \%$ ); carbapenems and glycopeptides were used in $4 \%$ and $3 \%$ of patients respectively. Most of the patients started the antimicrobial therapy intravenous $(97 \%)$ to switch to oral therapy just few times $(6 \%)$. The mean duration of antibiotic treatment in hospital was $9.5 \pm 6.1$ SD days. Moreover, $18 \%$ of patients continued the treatment at home for further 6 days on average. In Table 3 are reported the different empiric therapeutic approaches for CAIs, HAIs, HCAIs and not epidemiolog-

Table 2. Sites of infection.

\begin{tabular}{lcc}
\hline Site of infection & No. of patients & $\mathbf{\%}$ \\
\hline Respiratory tract & 214 & 44 \\
\hline Urinary tract & 68 & 14 \\
\hline Biliary tract & 24 & 4.9 \\
\hline Gastro-intestinal tract & 47 & 9.6 \\
\hline Skin and soft tissue & 39 & 8 \\
\hline Sepsis & 29 & 5.9 \\
\hline Others & 9 & 1.8 \\
\hline NA & 55 & 11.3 \\
\hline
\end{tabular}

NA, not available.

Table 3. Empiric therapeutic approach for community-acquired, hospital-acquired, health care-associated and not-epidemiologically-classified infections.

\begin{tabular}{|c|c|c|c|c|}
\hline & CAIs $(n=215)$ & HAIs $(n=43)$ & HCAIs $(n=50)$ & NECIs $(n=177)$ \\
\hline Ampicillin-sulbactam or amoxicillin-clavulanate & $71(33.0 \%)$ & $8(18.6 \%)$ & $6(12.0 \%)$ & $48(27.1 \%)$ \\
\hline Piperacillin-tazobactam & $39(18.1 \%)$ & $6(13.9 \%)$ & $21(42.0 \%)$ & $15(8.4 \%)$ \\
\hline Ceftriaxone & $38(17.6 \%)$ & $7(16.2 \%)$ & $6(12.0 \%)$ & $51(28.8 \%)$ \\
\hline Fluoroquinolones & $40(18.6 \%)$ & $9(20.9 \%)$ & $6(12.0 \%)$ & $37(20.9 \%)$ \\
\hline Carbapenems & - & $6(13.9 \%)$ & $4(8.0 \%)$ & - \\
\hline Vancomycin & - & $5(11.6 \%)$ & - & - \\
\hline Others & $27(12.5 \%)$ & $2(4.6 \%)$ & $7(14.0 \%)$ & $26(14.6 \%)$ \\
\hline Combination therapy & $37(17.2 \%)$ & $10(23.2 \%)$ & $21(42.0 \%)$ & $32(18.0 \%)$ \\
\hline
\end{tabular}

CAIs, community-acquired infections; HAIs, hospital-acquired infections; HCAIs, health care-associated infections; NECIs, not-epidemiologically-classified infections. 
ically classified infections (NECIs). CAIs were treated with ampicillin/sulbactam or amoxicillin/clavulanate (33\%), fluoroquinolones $(18.6 \%)$, piperacillin/tazobactam (18.1\%), ceftriaxone (17.6\%) and various other antimicrobials (12\%). Out of these, $37(17 \%)$ received a combination of two or more antibiotics. HAIs were treated with fluoroquinolones (20.9\%), ampicillin/sulbactam (18.6\%), ceftriaxone (16.2\%), piperacillin/ tazobactam (13.9\%), meropenem (13.9\%), and vancomycin (11.6\%); combination with another antibiotic (clarithromycin, fluoroquinolones, teicoplanin, gentamycin, metronidazole, colistin) was made in $10 \mathrm{pa}-$ tients $(23 \%)$. The antibiotic more frequently selected to treat HCAIs $(n=50)$ was piperacillin/tazobactam $(42 \%)$, followed by fluoroquinolones, ampicillin/sulbactam, ceftriaxone and metronidazole $(12 \%$ each $)$ and meropenem (8\%); combination regimens (using fluoroquinolones, glycopeptides, macrolide and aminoglycoside) were administered in 21 patients $(42 \%)$. For the treatment of NECIs $(n=177)$ were administered ceftriaxone (28.8\%), ampicillin/sulbactam or amoxicillin/ clavulanate (27.1\%), fluoroquinolones (20.9\%), piperacillin/tazobactam (8.4\%); combination regimens were used in 32 patients $(18 \%)$. As shown in Table 4, remarkable differences were observed comparing the data of the various centers. The rate of PUAT ranged from $13 \%$ to $75 \%$, the length of hospital stay from 7.8 $\pm 7.7 \mathrm{SD}$ days to $16.6 \pm 12 \mathrm{SD}$ days $(\mathrm{P}<0.001)$, the mean duration of antibiotic treatment in hospital from $6.8 \pm 4.3 \mathrm{SD}$ days to $11.4 \pm 7.8 \mathrm{SD}$ among the various hospitals $(\mathrm{P}<0.05)$.

\section{Discussion}

In our observational study, more than half (58.5\%) of patients admitted to the Internal Medicine wards received antibiotic therapy. The percentage significantly differed in different centers, ranging from $13 \%$ to $71 \%$. These data are representative of the heterogeneity among Internal Medicine units and confirm several previous reports. Cusini et al. reported in Switzerland a mean prevalence of $44 \%$ among all hospital departments (including surgical and medical areas), with a rate of $46 \%$ for medical wards; hematology-oncology wards reached the higher value $(57 \%){ }^{1}$ The European Surveillance of Antimicrobial Consumption (ESAC) showed a mean prevalence of $30 \%$, even if ranging from $19 \%$ to $59 \%$ between 20 European hospitals. Several other studies conducted during the period 19992008 found the rate of patients receiving antibiotics ranging between 16 to $49 \%$, but data were based on different departments, pediatric included, and not only on Internal Medicine wards. ${ }^{6-14}$ Although the number of patients treated with antibiotic in our Internal Medicine wards seems exceedingly high, a more specific comparison in terms of departments, patients, diseases and comorbidities should be useful to evaluate any antimicrobial misuse. However, our results seem to show some important understatements in terms of antibiotic stewardship. Despite it is well recognized that patients with hospital acquired and health-care associated infections should be empirically treated with antibiotics active against specific multidrug resistant pathogens (i.e., Pseudomonas spp. and methicillin resistant S. aureus), one third of the patients in our series did not receive any epidemiological classification. As a consequence, patients with CAI could have been treated with unnecessary broad-spectrum drugs and, otherwise, patients with HAI could have been undertreated. Our data actually show that almost twenty percent of patients with CAI received a treatment with antibiotics appropriate for HAI (e.g., piperacillin-tazobactam) and probably unnecessary. Conversely, more than half of HAIs and HCAIs received treatments, which should be reserved to CAIs, frequently ( $80 \%$ of cases) using a single agent.

Table 4. Comparison of different Hospitals.

\begin{tabular}{|c|c|c|c|}
\hline Hospital & $\begin{array}{c}\text { PUAT } \\
(\% \text { total })\end{array}$ & $\begin{array}{l}\text { Duration of therapy } \\
\quad(\text { days } \pm \text { SD) }\end{array}$ & $\begin{array}{l}\text { Length of stay } \\
\quad \text { (days } \pm \text { SD) }\end{array}$ \\
\hline All centers & 58.5 & $9.5 \pm 6.1$ & $13.7 \pm 10.4$ \\
\hline San Giovanni - Roma & 55 & $8.2 \pm 5.7$ & $11.2 \pm 8.9$ \\
\hline Sant'Eugenio - Roma & 60 & $10.1 \pm 4.6$ & $10.1 \pm 5.6$ \\
\hline Vannini - Roma & 58 & $9.7 \pm 6.1$ & $13.7 \pm 10.4$ \\
\hline Sandro Pertini - Roma & 71 & $11.4 \pm 7.8$ & $16.6 \pm 12$ \\
\hline Fatebenefratelli Isola Tiberina - Roma & 75 & $9.7 \pm 6$ & $11.6 \pm 9.3$ \\
\hline L. Parodi Delfino - Colleferro & 60 & $8.2 \pm 4.9$ & $10.8 \pm 6.9$ \\
\hline San Camillo de Lellis - Rieti & 13 & $6.8 \pm 4.3$ & $7.8 \pm 7.7$ \\
\hline Montefiascone & 50 & $9.2 \pm 1.7$ & $12.5 \pm 5.2$ \\
\hline Santa Maria Goretti - Latina & 50 & $7.4 \pm 2.7$ & $8.5 \pm 8.2$ \\
\hline
\end{tabular}

PUAT, patients under antimicrobial treatment; SD, standard deviation. 
This under treatment exposes these patients to higher risk of failure (complications and/or death). Overall, the selected antimicrobials were considered inadequate in $30 \%$ of cases according to the epidemiological classification received. Moreover, microbiological diagnosis was attempted in less than half of patients in the PUAT group and was obtained in 50\% of cultured patients; therefore, only $25 \%$ of all PUAT received an etiologic diagnosis. This can explain why almost all treatments were empiric and so few targeted (5\%). In a multicenter interventional study, $27.3 \%$ of antibiotic courses were determined to be unjustified; a post-prescription intervention based on an audit and feedback approach in addition to current stewardship programs, lead the clinicians to accept recommendations to change or stop the antibiotics in $66.7 \%$ of cases..$^{15}$ Compared to this trial, the inappropriateness of antibiotic prescription observed in our Internal Medicine wards was not so different. Excessive or inappropriate antimicrobial use increases morbidity, mortality, and healthcare costs including prolonged hospitalization. ${ }^{7-11}$ Our results might be in accordance with these premises because PUAT showed a significantly higher mortality and prolonged length of stay. However, PUAT were also significantly older then PWT and the higher mortality, as well as the prolonged length of stay, could also be attributable to comorbidities and lower performance status, rather than to the antibiotic misuse. In the attempt to prevent the development of $C$. difficile infections, the antibiotic stewardship program guidelines by the Infectious Diseases Society of America (IDSA)/Society for Healthcare Epidemiology of America (SHEA) highlight as a priority to reduce the use of high-risk antibiotics such as clindamycin, $2^{\text {nd }}$ and $3^{\text {rd }}$ generation cephalosporins and fluoroquinolones. ${ }^{16-18}$ In our study, protected penicillins were the antibiotic of choice in almost half of the cases, but $3^{\text {rd }}$ generation cephalosporins and fluoroquinolones still accounted for $45 \%$ of cases overall. Interventions to reduce and minimize the consumption of these high-risk antibiotics should be a major challenge. It is not surprising that almost all patients admitted to Internal Medicine wards have antibiotics administered for intravenous route. However, the early switch to oral therapy has several advantages such as reduced risk of cannula-related infections, reduced risk of thrombophlebitis, lower costs, reduction of hidden costs (cost of diluents, needles, syringes, nursing time, etc.) and earlier discharge. For all these reasons intravenous to oral switch is strongly advocated in clinically stable patients. In our series the switch occurred in only $6 \%$ of patients. Based on these data, an intervention addressed to this topic probably should be included in the local stewardship programs. Antibiotic consumption is strongly related to duration of therapy. In the last few years, several studies documented similar efficacy of short-course regimens compared to standard regimens.
Antibiotic stewardship interventions aimed at reducing the duration of antibiotic therapy lead to similar clinical outcomes compared to pre-intervention period. Specifically, 7 and 10 days of treatment for inpatients with community acquired pneumonia were equally effective in terms of clinical outcome, length of stay and 30-day readmission rates. ${ }^{19,20}$ Similarly, for patients with ventilator associated pneumonia, 8-10 days of treatment have been proposed in substitution of the usual two weeks course $\mathrm{e}^{21-23}$ and the same proposal has been made for patients with cellulitis, pyelonephritis and intra-abdominal infection. ${ }^{24-28}$ Moreover, IDSA/SHEA guidelines suggest using inflammatory biomarker as PCT to shorten antimicrobial exposure, especially in intensive care units. Even if PCT was measured only in $26 \%$ of PUAT and therefore underutilized, the duration of antibiotic treatment in our center was not far from the numbers highlighted in the previous studies, with a mean length among various hospitals of $9.5 \pm 6.1 \mathrm{SD}$ days ranging from $6.8 \pm 4.3 \mathrm{SD}$ days to $11.4 \pm 7.8 \mathrm{SD}$ $(\mathrm{P}<0.05)$. Unfortunately, we were not able to relate the duration of treatment with the clinical outcome. It seems remarkable that around $18 \%$ of patients continued the treatment at home for further 6 days on average probably extending too long the antibiotic course.

\section{Limitations}

Our study has several limitations. The center responsible to collect all data records and to create a single database did not have any control over the other hospitals doctors and even if it was clearly highlighted the importance of scheduling every patient discharged during the recruitment period we are not sure if any was missed and therefore if a collecting bias is present. Not all centers recruited the same number of patients and the use of inflammatory markers (e.g., PCT) was very heterogeneous. The records were not always completely filled in and several info lacked. We did not evaluate comorbidities (e.g., Charlson comorbidity index), and complications related to antibiotic use (e.g., C. difficile infections, allergic reaction, acute kidney injury, etc.). The diagnosis made was not always clear and this did not allow us to properly evaluate the expected duration of therapy. Since it was not possible to estimate for every patient the diagnosis along with illness severity and associated comorbidities, comparing mortality and length of stay of PUAT vs PWT was not feasible.

\section{Conclusions}

Among patients admitted to Internal Medicine wards, more than half received antibiotic therapy. Compared to previous studies, this rate is probably too high. Moreover, a great variability was observed among several wards. Almost all patients were treated 
Table 5. Critical issues and possible corrective measure.

\begin{tabular}{|c|c|c|}
\hline & Reasons & Corrective measures \\
\hline \multirow[t]{5}{*}{$\begin{array}{l}\text { Excessive consumption/excessive } \\
\text { duration }\end{array}$} & Diagnosis questionable & $\begin{array}{l}\text { Make efforts to better define diagnosis and make an } \\
\text { epidemiological classification; do not treat colonized } \\
\text { asymptomatic patients }\end{array}$ \\
\hline & Poor guidelines adherence & Increase guidelines adherence, prefer short-course AT if advocate \\
\hline & Wrong antibiotic use & $\begin{array}{l}\text { Seek for an etiologic diagnosis, antimicrobial prophylaxis only in } \\
\text { selected patients }\end{array}$ \\
\hline & Low use of biomarkers & Use PCT to guide AT \\
\hline & $\begin{array}{l}\text { Antimicrobial stewardship } \\
\text { programs not considered }\end{array}$ & $\begin{array}{l}\text { Infectious disease specialist consultation, control on prescription, } \\
\text { de-escalation therapy, switch-therapy, route of administration } \\
\text { (prefer per os over i.v.) }\end{array}$ \\
\hline \multirow[t]{3}{*}{ Missed etiologic diagnosis } & Sample not collected & $\begin{array}{l}\text { Collect cultural samples at least once at first episode of fever or } \\
\text { suspicious of infection }\end{array}$ \\
\hline & Collection under AT & Collect cultural samples before starting AT \\
\hline & $\begin{array}{l}\text { Type of pathology/type of } \\
\text { cultural sample }\end{array}$ & $\begin{array}{l}\text { Collect culture from primary site of infection, blood culture always } \\
\text { useful }\end{array}$ \\
\hline \multirow[t]{3}{*}{ Clostridium difficile } & AT not needed & See: Excessive consumption/excessive duration corrective measure \\
\hline & Excessive duration & See: Excessive consumption/excessive duration corrective measure \\
\hline & Frail patients & $\begin{array}{l}\text { Isolate patients with } C \text {. difficile in single room } \\
\text { Use gloves } \\
\text { Wash hand between patients contact }\end{array}$ \\
\hline \multirow[t]{2}{*}{ Biomarkers } & Low availability & Increase availability and educate professional workers \\
\hline & Poor awareness of their use & Use PCT level to start or stop AT \\
\hline
\end{tabular}

AT, antibiotic therapy; PCT, procalcitonin; os, oral somministration; i.v., intravenous.

empirically, but one third did not receive any epidemiological classification, with the possible consequence of being under- or over-treated. Greater attention to the epidemiological classification should be recommended to drive the antibiotic choice. Switching from intravenous to oral antibiotic is still far from being a reality in Lazio, only $6 \%$ of patients belong to this group. According to the current trends to short-course regimen for most infections, the mean duration of 9.5 days, seems too long. Furthermore, many patients were discharged with prescription of antibiotics at home for almost another week. Inflammatory markers as PCT are not yet commonly used to reduce antibiotic utilization. Critical issues and possible corrective measure highlighted by this study have been summarized in Table 5. Antibiotic stewardship programs represent a primary objective to implement in the Internal Medicine wards of Lazio.

\section{References}

1. Cusini A, Rampini SK, Bansal V, et al. Different patterns of inappropriate antimicrobial use in surgical and medical units at a tertiary care hospital in Switzerland: a prevalence survey. PLoS One 2010;5:e14011.

2. Giannella M, Tedeschi S, Bartoletti M, Viale P. Prevention of infections in nursing homes: antibiotic prophylaxis versus infection control and antimicrobial stewardship measures. Expert Rev Anti Infect Ther 2016;14:219-30.

3. Schulte DM, Sethi A, Gangnon R, et al. Risk factors for Candida colonization and Co-colonization with multidrug resistant organisms at admission. Antimicrob Resist Infect Control 2015;4:46.

4. Raineri E, Pan A, Mondello P, et al. Role of the infectious diseases specialist consultant on the appropriateness of antimicrobial therapy prescription in an intensive care unit. Am J Infect Control 2008;36:283-90.

5. Lai CC, Shi ZY, Chen YH, Wang FD. Effects of various antimicrobial stewardship programs on antimicrobial usage and resistance among common gram-negative bacilli causing health care-associated infections: a multicenter comparison. J Microbiol Immunol Infect 2016; 49:74-82.

6. Ansari F, Erntell M, Goossens H, Davey P. The European Surveillance of Antimicrobials Consumption (ESAC) point-prevalence survey of antibacterial use in 20 European Hospitals in 2006. Clin Infect Dis 2009;49: 1496-504.

7. Paterson DL. "Collateral damage" from cephalosporin or quinolone antibiotic therapy, Clin Infect Dis 2004;38: S341-5.

8. Albrich WC, Monnet DL, Harbarth S. Antibiotic selection pressure and resistance in Streptococcus pneumoniae and Streptococcus pyogenes. Emerg Infect Dis 2004;10:514-7. 
9. Goossens H, Ferech M, Vander Stichele R, et al. Outpatient antibiotic use in Europe and association with resistance: a cross-national database study. Lancet 2005; 365:579-87.

10. Malhotra-Kumar S, Lammens C, Coenen S, et al. Effect of azithromycin and clarithromycin therapy on pharyngeal carriage of macrolide-resistant streptococci in healthy volunteers: a randomised, double blind, placebocontrolled study. Lancet 2007;369:482-90.

11. Lodise TP, McKinnon PS. Clinical and economic impact of methicillin resistance in patients with Staphylococcus aureus bacteremia. Diagn Microbiol Infect Dis 2005;52: 113-22.

12. Lee NY, Lee HC, Ko NY, et al. Clinical and economic impact of multidrug resistance in nosocomial Acinetobacter baumannii bacteremia. Infect Control Hosp Epidemiol 2007;28:713-9.

13. Venditti M, Falcone M, Corrao S, et al. Outcomes of patients hospitalized with community-acquired, health care-associated, and hospital-acquired pneumonia. Ann Intern Med 2009;150:19-26.

14. American Thoracic Society; Infectious Diseases Society of America. Guidelines for the management of adults with hospital-acquired, ventilator-associated, and healthcare-associated pneumonia. Am J Respir Crit Care Med 2005;171:388-416.

15. Cosgrove SE, Seo SK, Bolon MK, et al. Evaluation of postprescription review and feedback as a method of promoting rational antimicrobial use: a multicenter intervention. Infect Control Hosp Epidemiol 2012;33:374-80.

16. Barlam TF, Cosgrove SE, Abbo LM, et al. Implementing an antibiotic stewardship program: guidelines by the Infectious Diseases Society of America and the Society for Healthcare Epidemiology of America. Clin Infect Dis 2016;62:e51-77.

17. Cyriac JM, James E. Switch over from intravenous to oral therapy: a concise overview. J Pharmacol Pharmacother 2014;5:83-7.

18. Fischer MA, Solomon DH, Teich JM, Avorn J. Conversion from intravenous to oral medications: assessment of a computerized intervention for hospitalized patients. Arch Intern Med 2003;163:2585-9.
19. Dimopoulos G, Matthaiou DK, Karageorgopoulos DE, et al. Short- versus long-course antibacterial therapy for community-acquired pneumonia: a meta-analysis. Drugs 2008;68:1841-54.

20. Avdic E, Cushinotto LA, Hughes AH, et al. Impact of an antimicrobial stewardship intervention on shortening the duration of therapy for community-acquired pneumonia. Clin Infect Dis 2012;54:1581-7.

21. Pugh R, Grant C, Cooke RP, Dempsey G. Short-course versus prolonged-course antibiotic therapy for hospitalacquired pneumonia in critically ill adults. Cochrane Database Syst Rev 2011;(1):CD007577.

22. Dimopoulos G, Poulakou G, Pneumatikos IA, et al. Short-versus long-duration antibiotic regimens for ventilatorassociated pneumonia: a systematic review and meta-analysis. Chest 2013;144:1759-67.

23. Chastre J, Wolff M, Fagon JY, et al. Comparison of 8 vs 15 days of antibiotic therapy for ventilator-associated pneumonia in adults: a randomized trial. JAMA 2003;290:2588-98.

24. Hepburn MJ, Dooley DP, Skidmore PJ, et al. Comparison of short-course ( 5 days) and standard (10 days) treatment for uncomplicated cellulitis. Arch Intern Med 2004;164:1669-74.

25. Sandberg T, Skoog G, Hermansson AB, et al. Ciprofloxacin for 7 days versus 14 days in women with acute pyelonephritis: a randomised, open-label and doubleblind, placebo-controlled, non-inferiority trial. Lancet 2012;380:484-90.

26. Talan DA, Stamm WE, Hooton TM, et al. Comparison of ciprofloxacin (7 days) and trimethoprim-sulfamethoxazole (14 days) for acute uncomplicated pyelonephritis pyelonephritis in women: a randomized trial. JAMA 2000;283:1583-90.

27. Runyon BA, McHutchison JG, Antillon MR, et al. Shortcourse versus long-course antibiotic treatment of spontaneous bacterial peritonitis. A randomized controlled study of 100 patients. Gastroenterology 1991;100:1737-42.

28. Sawyer RG, Claridge JA, Nathens AB, et al. Trial of short-course antimicrobial therapy for intraabdominal infection. N Engl J Med 2015;372:1996-2005. 